\title{
NUMERICAL SIMULATION OF THE MOLTEN POOL OF A POWDER BED
}

\author{
Y. Saadlaoui ${ }^{1}$, E. Feulvarch ${ }^{1}$, J-B. Leblond ${ }^{2}$ and J-M. Bergheau ${ }^{1}$ \\ ${ }^{1}$ University of Lyon, CENTRALELYON-ENISE, LTDS, CNRS, UMR 5513. \\ 58 rue Jean Parot, 42023 Saint-Etienne Cedex 02, France, \\ yassine.saadlaoui@enise.fr, eric.feulvarch@enise.fr, jean-michel.bergheau@enise.fr \\ ${ }^{2}$ University of Sorbonne, Institut Jean Le Rond d'Alembert, CNRS, UMR7190 \\ 4 place Jussieu, 75005 Paris, France \\ jbl@lmm.jussieu.fr
}

Key words: Molten pool, fluid flows, Surface tension, Numerical simulation, Melting of a powder bed.

\begin{abstract}
In this study, a numerical approach is developed to simulate the molten pool formation during the melting of a powder bed. It is based on a fluid formulation that allows taking into account the dynamics in the molten pool through the two effects of surface tension (including both "curvature effect" and the "Marangoni effect") and buoyancy. Additionally, the free surface is considered using an ALE method. The shrinkage of the powder layer after its melting and the change of the thermo-physical properties depending on the material state (powder or compact) are also modeled. As an application, a 3D thermo-fluid simulation of the powder bed melting is carried out. It is found that the powder layer porosity has a great effect on the molten pool morphology.
\end{abstract}

\section{INTRODUCTION}

Additive manufacturing processes have begun to occupy an increasingly important place in the industrial world, especially those based on the melting of a powder bed. They consist of producing parts through layer by layer deposition and selective melting of powder layers using a laser. During the melting of a powder bed, different materials can be used: polymers, metals, and ceramics. Metals are by far the most used materials in different industrial environments such as medical, industry energy, aerospace, and automotive. The melting of a powder bed requires continuous studying to improve its performance. Experimental approaches are often used to study and improve this process [1]. These approaches can be very expensive and inaccessible. For this, the numerical simulation can be an alternative solution. It can allow optimizing the process parameters for a reasonable cost and time.

Depending on the objectives targeted by the simulation, different numerical approaches have been developed. These approaches can be classified according to the simulation scale: powder scale (microscopic scale), molten pool scale (mesoscopic scale), and part scale (macroscopic scale). The scale can be changed according to the aim of the numerical simulation. For example, the aim of the macroscopic simulation is to estimate the residual stresses and distortions, thus providing very useful information for fatigue lifetime predictions. The thermal part of this simulation kind is generally simplified in order 
to reduce the computation time since it is used to simulate large structures. This type of simulation is not totally predictive as far as temperature distributions are concerned. Their prediction indeed requires carefully simulating the fluid flows, which plays an important role, particularly on the morphology of the molten pool [2]. These fluid flows are generally taken into account during the microscopic and mesoscopic simulations. Indeed, the aim of these two simulations is to consider the physical phenomena involved by the process itself [2], [3]. However, the complexity of the modeling of these phenomena makes the computation time very important especially for the microscopic simulation [4], [5], [6].

In this context, this study proposes a new approach to simulate the melting of a powder bed at the molten pool scale. It is based on the formulation of the fluid problem developed by Saadlaoui et al. [2]. This formulation allows taking into account the surface tension (including both the "curvature effect" and the "Marangoni effect" [7]), buoyancy forces, and the free surface evolution. The originality of this formulation remains its simplicity, its efficiency, and especially its computation time, which is very reasonable [2]. Thus, the proposed approach allows taking into account the dynamics in the molten pool, the change of the thermo-physical properties depending on the material state (powder or compact), and the shrinkage of the powder layer after its melting. The paper is organized as follows:

- in Section 2, the proposed approach to simulate the melting of a powder bed at the molten pool scale is presented. It contains three parts: presentation of some physical phenomena involved during this process, fluid formulation, and the procedure of the proposed approach.

- in Section 3, a 3D thermo-fluid simulation of the melting of a powder bed is presented. It will allow us to study the effect of the powder layer porosity on numerical results (fluid velocity, free
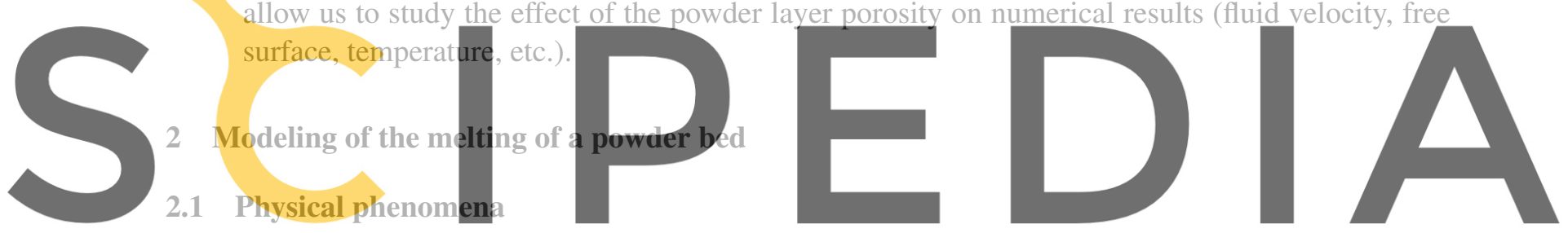

Regist The powder bed melting process.is a thermo-mechanical process that shares several physical phenomena Register for free at https/.WwW. scipedia.com to dowhload the version without the watermark

techniques. However, this process differs in a few points. Thus, during the melting of a powder bed, the raw material is in the form of the granular media. This media has completely different properties from those of a solid part (continuous media):

- Density: for the same material, the powder layer is a granular media that has a lower density than that of a compact media (solid part). This difference is related to the high porosity in a powder layer. This porosity decreases and then can disappear after the melting. This can cause an increase in density and thus shrinkage of the layer.

- Thermal conductivity: a powder layer has a lower thermal conductivity than that of a solid part of the same material. This is related to the powder layer porosity.

- Specific heat: because of its porosity, a powder layer has a different specific heat than that of a solid part of the same material. 
It can be necessary to take into account these phenomena, and especially the powder layer shrinkage during the numerical simulation of the powder bed melting. Controlling the shrinkage of the powder layer can help avoid manufacturing defects such as porosity formation into manufactured parts. Indeed, as shown by figures 1 and 2, the shrinkage of the first powder layer (thickness $e$ ) can generate the increasing of the second layer thickness (thickness $e+\Delta e$ ). Machine parameters initially optimized according to the initial thickness $e$ can be inadequate to melt a thickness of $e+\Delta e$. The unfused powder into manufactured parts can generate porosity which will affect the quality of these parts. Moreover, the thermal properties of a material such as thermal conductivity and specific heat have a direct effect on the temperature evolution and the molten pool morphology. Therefore, well-defined properties during the simulation can be necessary to ensure good numerical results.

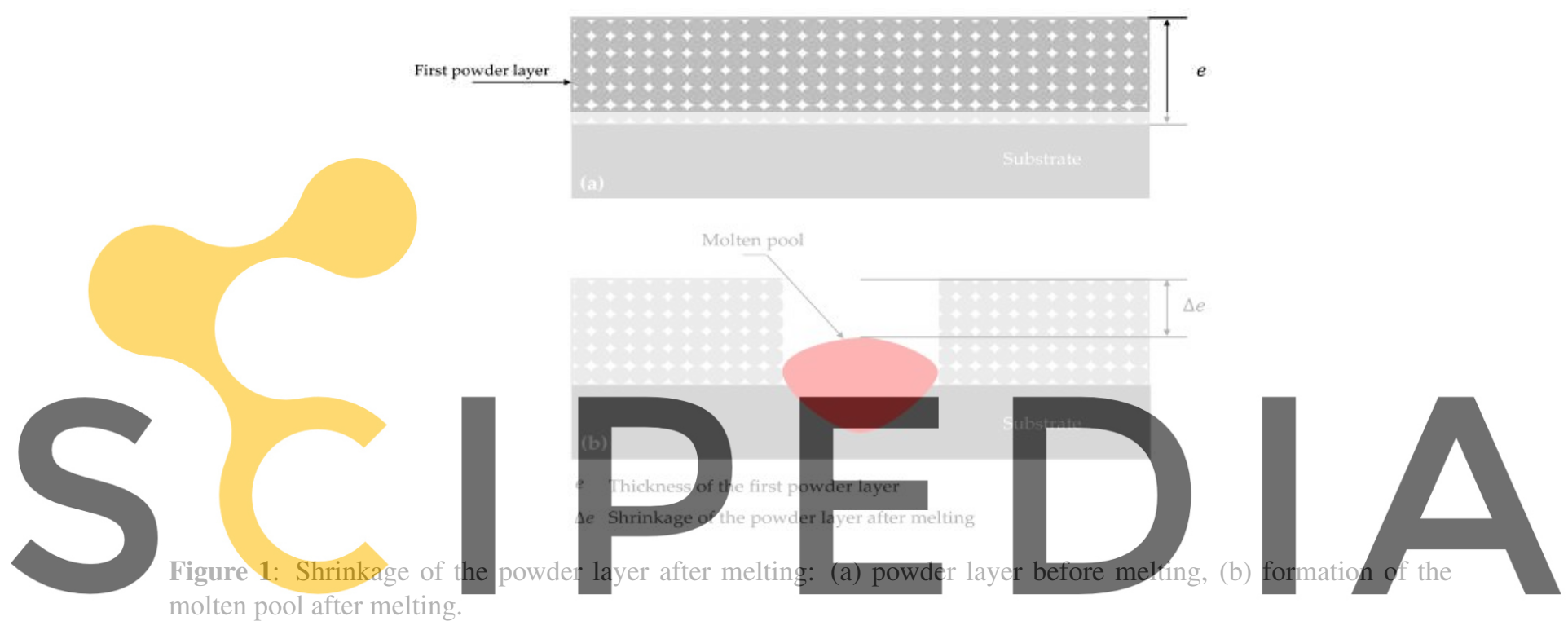

Register for free at https//www.scipedia.com to download the version without the watermark

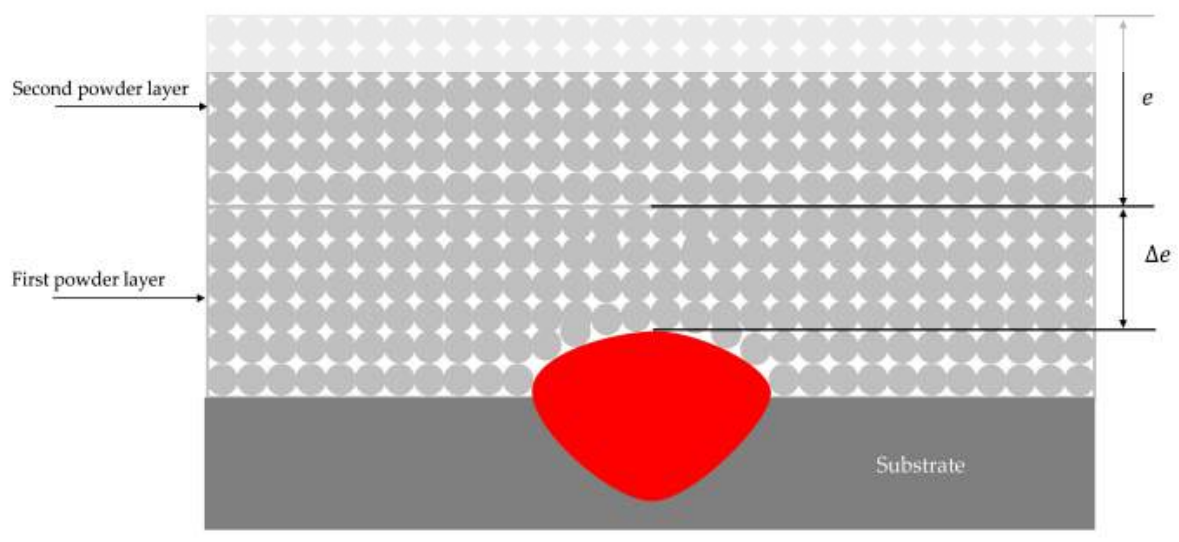

$e+\Delta e$ Thickness to melt

Figure 2: New thickness of the second powder layer. 


\subsection{Fluid formulation}

The fluid formulation developed by Saadlaoui et al. [2] is used to take into account the formation of the molten pool. The momentum equations can be written in the following form:

$$
-\operatorname{grad} p+\operatorname{divs}+\mathbf{f}_{\mathbf{v}}=\rho\left(\frac{\partial \mathbf{v}}{\partial t}+\operatorname{grad} \mathbf{v} . \mathbf{v}\right)
$$

The fluid flow is supposed to be laminar and Newtonian. Here $p$ is the fluid pressure, $\mathbf{s}$, the stress deviator, $\mathbf{f}_{\mathbf{v}}$, the volume force, and $\mathbf{v}$, the fluid velocity. Buoyancy forces can be considered using the volume forces which are given by the following equation:

$$
\mathbf{f}_{\mathbf{v}}=\rho_{0} \mathbf{g}\left(1-\beta\left(T-T_{0}\right)\right)
$$

$\mathrm{g}$ being the gravitational acceleration and $\rho_{0}$ the mass per unit volume at the reference temperature $T_{0}$. The finite element formulation of the fluid problem relies on a mixed velocity-pressure $(\mathbf{v}, p)$ variational formulation. The problem is now to find $(\mathbf{v}, p) \in\left(v_{a d} \times P_{a d}\right)$ such as $\forall\left(\mathbf{v}^{*}, p^{*}\right) \in\left(v_{a d}^{0} \times P_{a d}\right)$ :
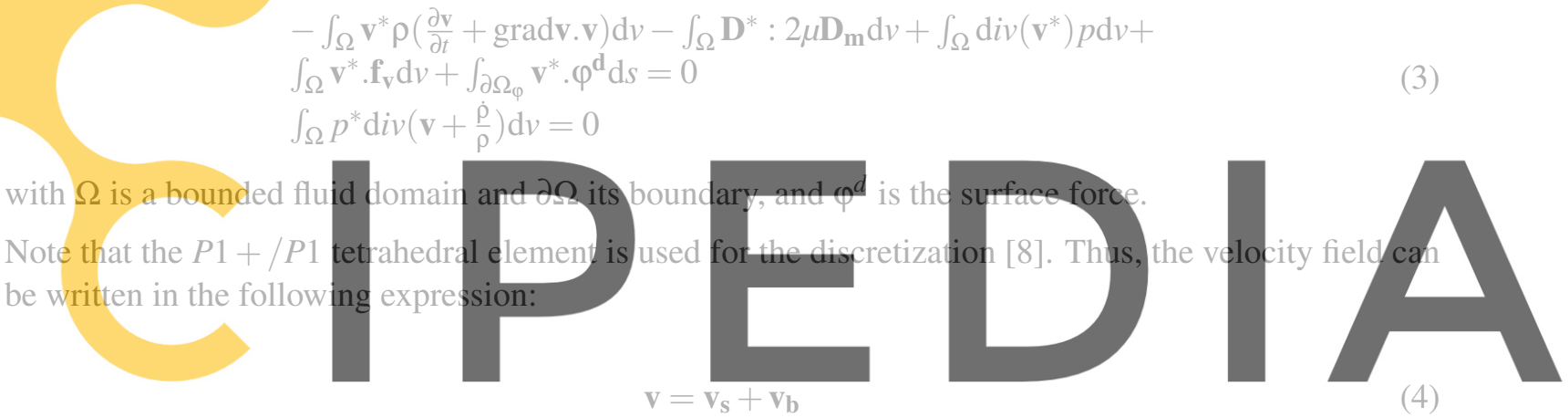

$\mathbf{v}=\mathbf{v}_{\mathrm{s}}+\mathbf{v}_{\mathrm{b}}$

(4)

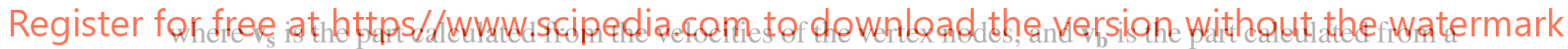

vector of additional degrees of the freedom of the bubble (internal) node. An implicit backward scheme for $\frac{\partial v}{\partial t}$ and a linearization of the advection term (gradv.v) are used for the time integration. This allows linearizing the fluid problem in order to minimize the computation time [2].

The surface tension is modeled through a surface hydrostatic state of stress $\tau=\left[\begin{array}{ll}\gamma & 0 \\ 0 & \gamma\end{array}\right]$, where $\gamma$ is the surface tension, applied to membrane-type elements (skin elements) that do not have any material properties [7]. The weak formulation of the fluid problem must be modified by adding the term $-\int_{\partial \Omega_{\text {free }}} \mathbf{D}_{\mathbf{s}}^{*}: \tau \mathrm{d} s$ to take into account at the same time the two effects of the surface tension, where $\partial \Omega_{\text {free }}$ is the free surface of the fluid.

\subsection{Proposed approach}

In this section, the principle, as well as the steps for implementing the proposed approach, are presented. The principle of this approach is to assume that the powder layer is a continuous media that has characteristics different from those of a solid part. Thus, the material is considered as a layer of powder 
(state 1) before the melting. Once the powder melted, it becomes a compact material (state 2) which has other properties. The different steps of the approach implementation are given by figure 3 . As shown in figure 3 , the initial mesh is assumed to consist of a powder layer (state 1), which has a density $\rho_{p}$, a thermal conductivity $\lambda_{p}$, and a specific heat $C_{p}$. The first step consists in beginning with a purely thermal simulation to reach the melting temperature of the material. Then, we start the loop with a time step of the thermo-fluid computation by activating the fluid flows in the molten pool. The elements which have a temperature lower than the solidus temperature $T_{\text {solidus }}$ remain in state 1 (powder). Thus, nodes with temperatures lower than $T_{\text {solidus }}$ remain fixed (solid nodes). The powder layer begins to transform in state 2 between $T_{\text {solidus }}$ and $T_{\text {liquidus. }}$. The fraction of material transformed in state 2 remains definitively in state 2 . The properties of the state mixture $\rho_{m}, \lambda_{m}$, and $C_{m}$ are calculated by combining the properties of the two states (states 1 and 2). Thus, for $T$ between $T_{\text {solidus }}$ and $T_{\text {liquidus: }}$ :

$$
\rho_{m}=X \rho_{c}+(1-X) \rho_{p}
$$

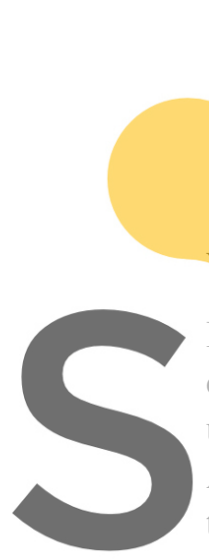

$$
\lambda_{m}=X \lambda_{c}+(1-X) \lambda_{p}
$$

$$
C_{m}=X C_{c}+(1-X) C_{p}
$$

is the fraction of state 2 ( $x=0$ for $T \leq T_{\text {solidus }}, x=1$ for $T \geq T_{\text {liquidus }}$ ).

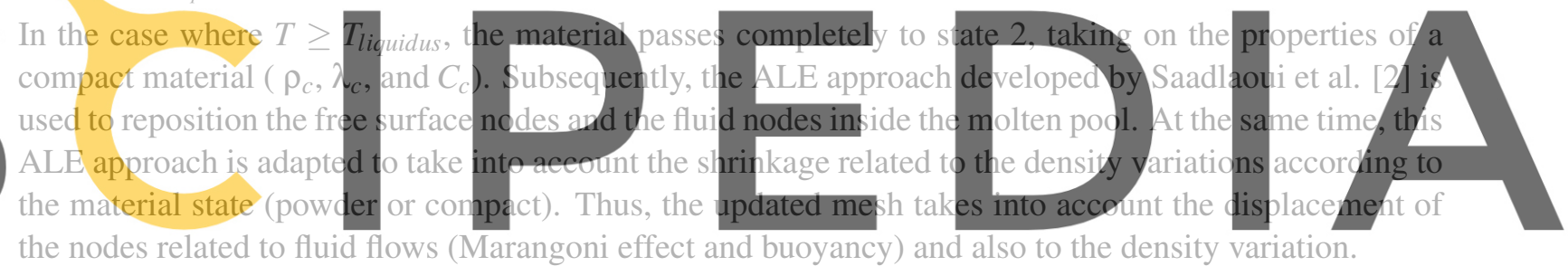

the nodes related to fluid flows (Marangoni effect and buoyancy) and also to the density variation.

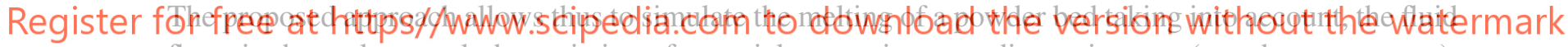
flows in the molten pool, the variation of material properties according to its state (powder or compact), and the shrinkage of the powder layer after its melting.

\section{Numerical simulation of the melting of powder bed process}

\subsection{FE model}

In this section, a 3D thermo-fluid simulation of the melting of a powder bed is presented. All simulations are performed in $S Y S W E L D^{T M}$ software. As shown in figure 4, the mesh consists of two blocks. The lower part (pink color) presents a 316L substrate with $8000 \mu m$ length, $500 \mu m$ width, and $500 \mu m$ thickness. A powder layer in $316 \mathrm{~L}$ (blue color) with $500 \mu \mathrm{m}$ thickness is initially present on the substrate. The thermo-physical properties of the powder layer (with a porosity of 20\%) and of the substrate are given in table 1 . The powder layer as well as the substrate have symmetry along the $Y Z$ plane. The mesh (figure 4) includes 120985 linear tetrahedral elements $(P 1+/ P 1), 22152$ linear triangular elements (skin elements), and 24701 nodes. In the powder bed as well as the substrate, the element size is $45 \mu \mathrm{m}$. 


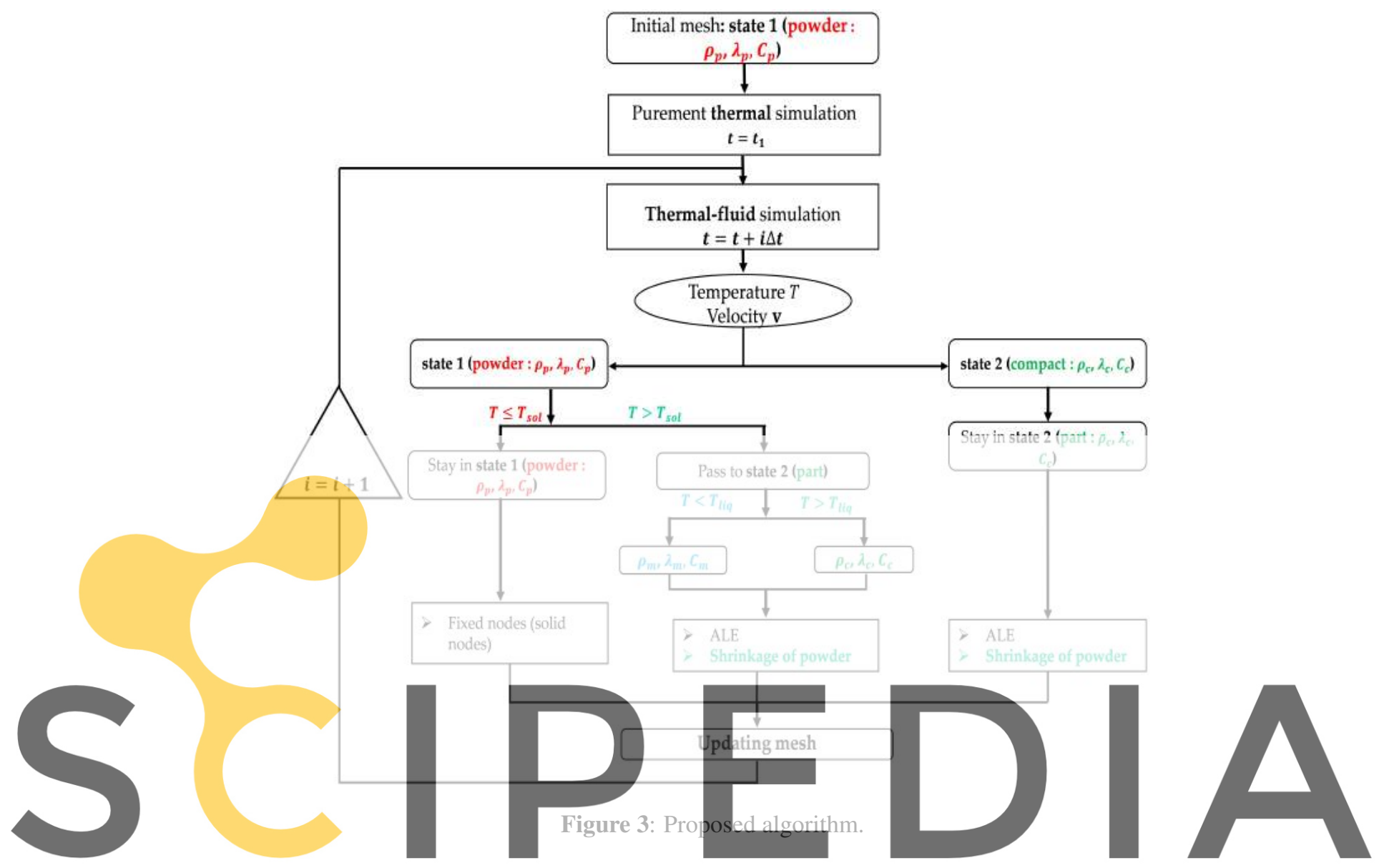

Register for free at https//www.scipedia.com to download the version without the watermark

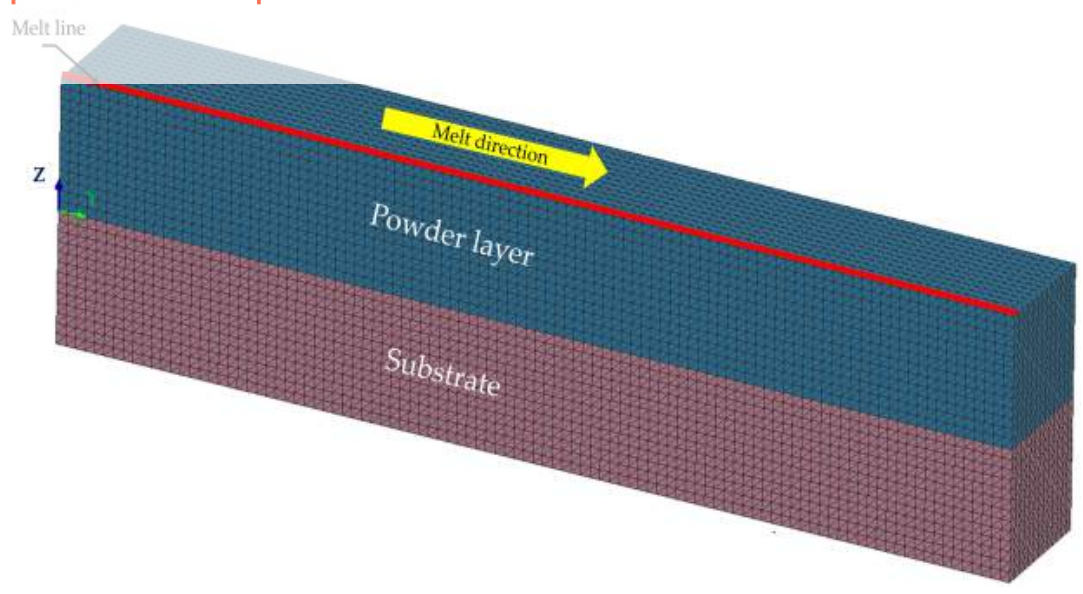

Figure 4: Mesh. 
An equivalent ellipsoidal heat source [3] [9] is applied in the melt direction to carry out a single track with a building speed of $500 \mathrm{~mm} \cdot \mathrm{s}^{-1}$. The laser power density of $6500 \mathrm{MW} \cdot \mathrm{m}^{-3}$ is applied (with a maximal front source intensity of 1600 and a maximal rear source intensity of 1500). It is assumed that $100 \%$ of the laser power is absorbed. A front length of $0.32 \mathrm{~mm}$, rear length of $0.5 \mathrm{~mm}$, half-width of $0.3 \mathrm{~mm}$, and penetration of $0.5 \mathrm{~mm}$ are used to define the heat source dimensions. The simulation time is $12 \mathrm{~ms}$, including $2 \mathrm{~ms}$ for the creation of the molten pool (thermal computation without fluid flows), and $10 \mathrm{~ms}$ for the thermo-fluid simulation (with fluid flows) with the motion of heat source. The time step is equal to $1 \mathrm{~ms}$ for the creation of the molten pool, and $10 \mu \mathrm{s}$ after the activation of the fluid flows. During the thermo-fluid simulation, in the solid zone ( $T \leq T_{\text {solidus }}$ ), the elements are assumed to have a high dynamic viscosity $\mu=10^{6} \mathrm{Kg} \cdot \mathrm{m}^{-1} \cdot \mathrm{s}^{-1}$. In the fluid zone, the elements have a dynamic viscosity equal to $\mu_{F}=6.210^{-2} \mathrm{Kg} \cdot \mathrm{m}^{-1} \cdot \mathrm{s}^{-1}$. A temperature-dependent surface tension $\gamma=\gamma_{L}+\alpha\left(T-T_{L}\right)$ with $\gamma_{L}=0.45 N \cdot m^{-1}, T_{L}=1500{ }^{\circ} \mathrm{C}$, and $\alpha=2 \cdot 10^{-4} N \cdot m^{-1} \cdot K^{-1}$ is taken into account to include both the "curvature" and "Marangoni" effects. Note that heat exchanges due to convection and radiation on the boundary with the external medium are taken into account:

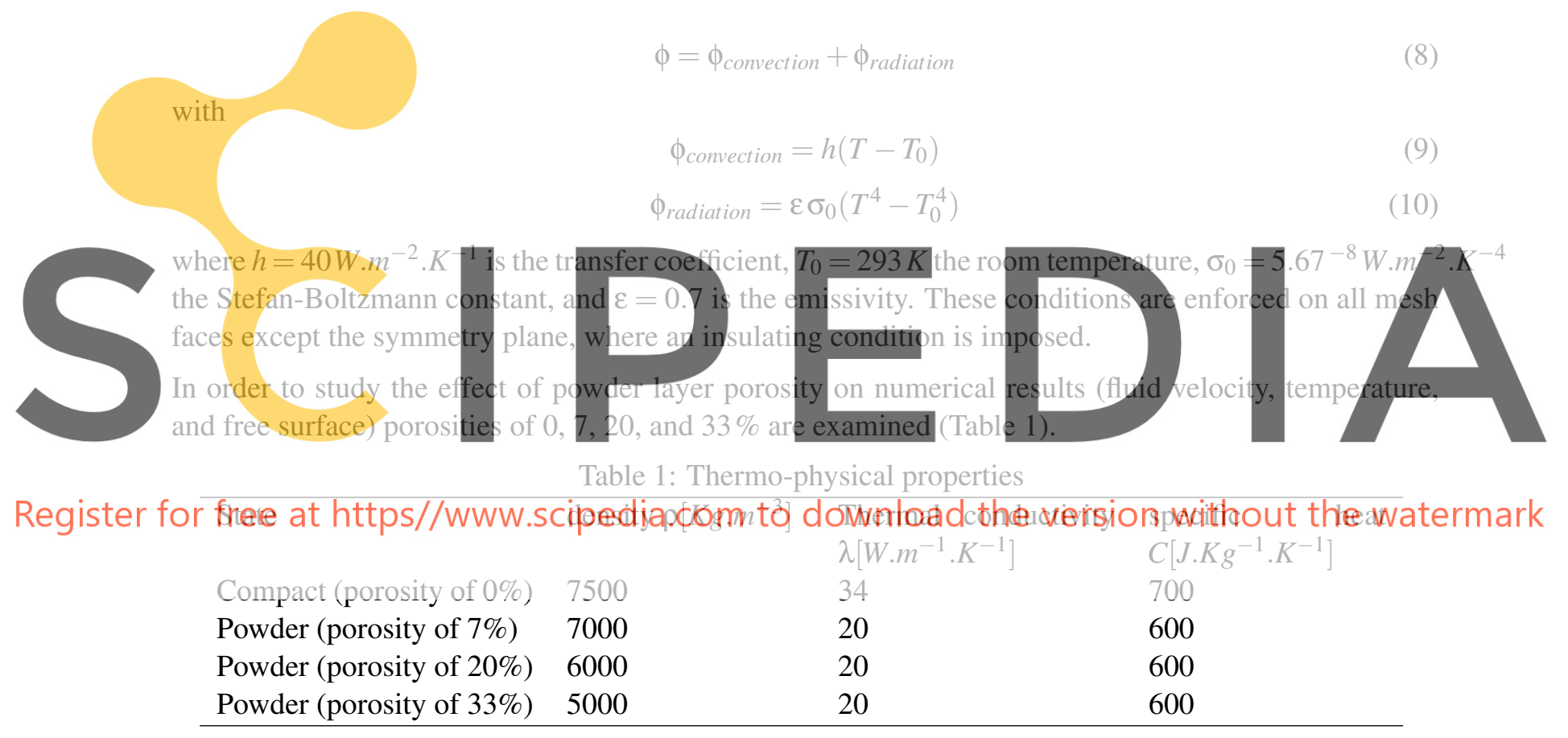

\subsection{Results and discussion}

In this section, the thermo-fluid results of the simulation of the powder bed melting are given. Figure 5 shows the distributions of the temperature and fluid velocity at instant $t=6 \mathrm{~ms}$. The fluid velocity is generated mainly by the Marangoni effect which is dominant during this process. This effect of a positive $\frac{\partial \gamma}{\partial T}$ is clearly visible in figure 5-b, it is linked to the temperature gradient. An average velocity approximately equal to $0.3 \mathrm{~m} . \mathrm{s}-1$ is found. This value is consistent with a literature values [2] [5] [6] [10]. 
Figure 6 shows the shrinkage of the powder layer $\Delta e$ after melting (displacement $U_{z}$ at $t=2.4 \mathrm{~s}$ ) for different porosity $(0,7,20$, and $33 \%)$. It is related to the density variation after the melting (transition from state 1 to state 2). As expected, this shrinkage is very sensitive to porosity. Indeed, it is more important for high porosities.

The porosity effects on temperature, flow velocity, and free surface are given respectively by figures 7 , 8 , and 9. Figure 7 shows that the free surface evolution is very sensitive to the porosity of the powder layer. A maximum gap of about $220 \%$ is found between the free surface displacements of porosities of 0 and $33 \%$. This great effect of the porosity is related to the powder layer shrinkage after its melting. This shrinkage has a direct effect on the free surface evolution and thus on the molten pool morphology. As shown in figure 7, the shrinkage is more important for high porosities. Thus, for example, the shrinkage is about $0.17 \mathrm{~mm}$ for a porosity of $33 \%$ whereas it is less than $0.05 \mathrm{~mm}$ for a porosity of $7 \%$. Contrary to the free surface, the fluid velocity in the molten pool is not very sensitive to porosity (figure 8). A maximum gap of about $12 \%$ is found between the velocities of porosities of 7 and $33 \%$. Similarly, the temperature is not very sensitive to the porosity of the powder layer. A maximum gap of about $7 \%$ is found between the temperatures of porosities of 7 and $33 \%$.
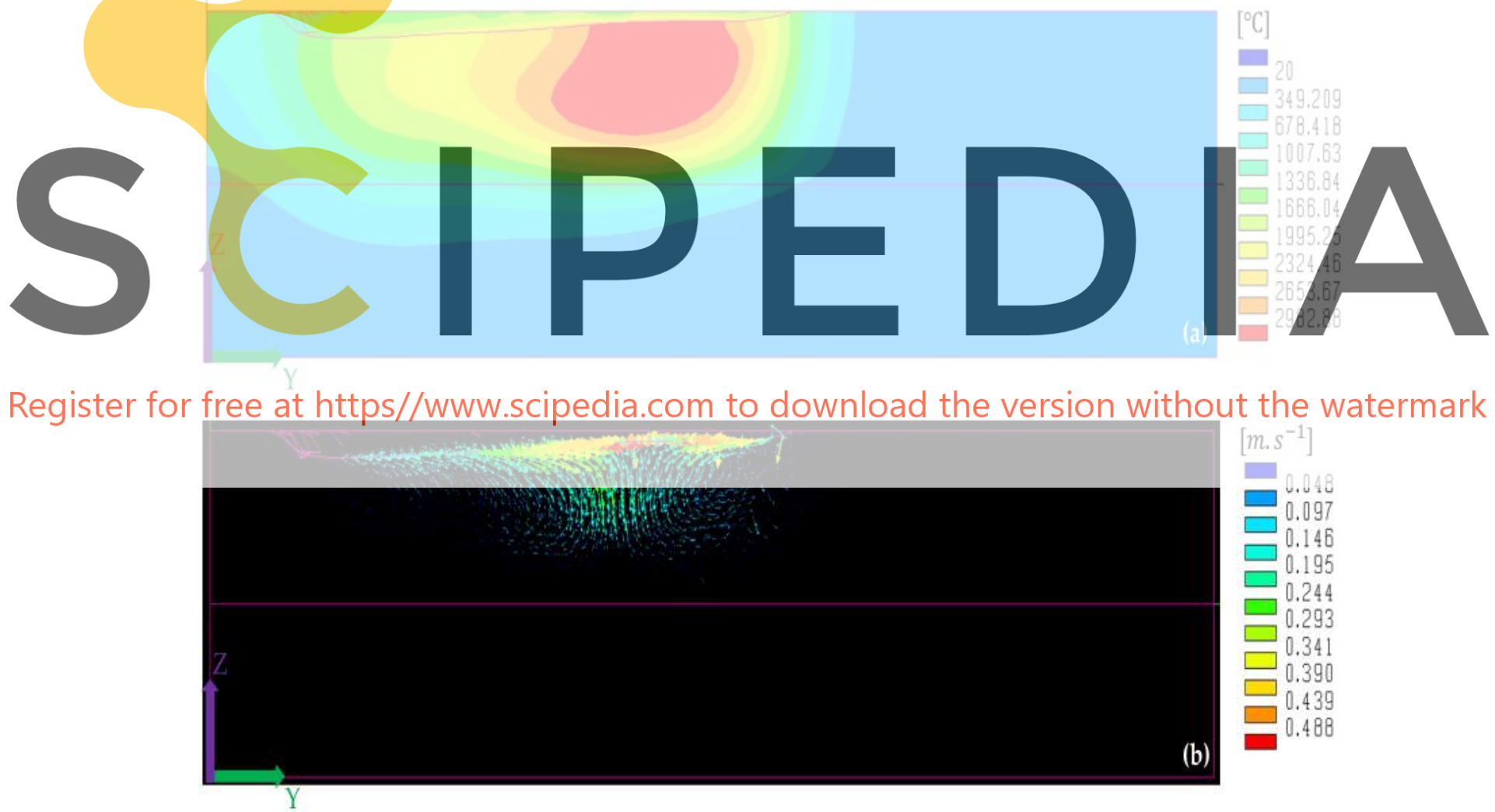

Figure 5: Thermo-fluid results (porosity of 20\%): (a) temperature field, (b) fluid velocity. 

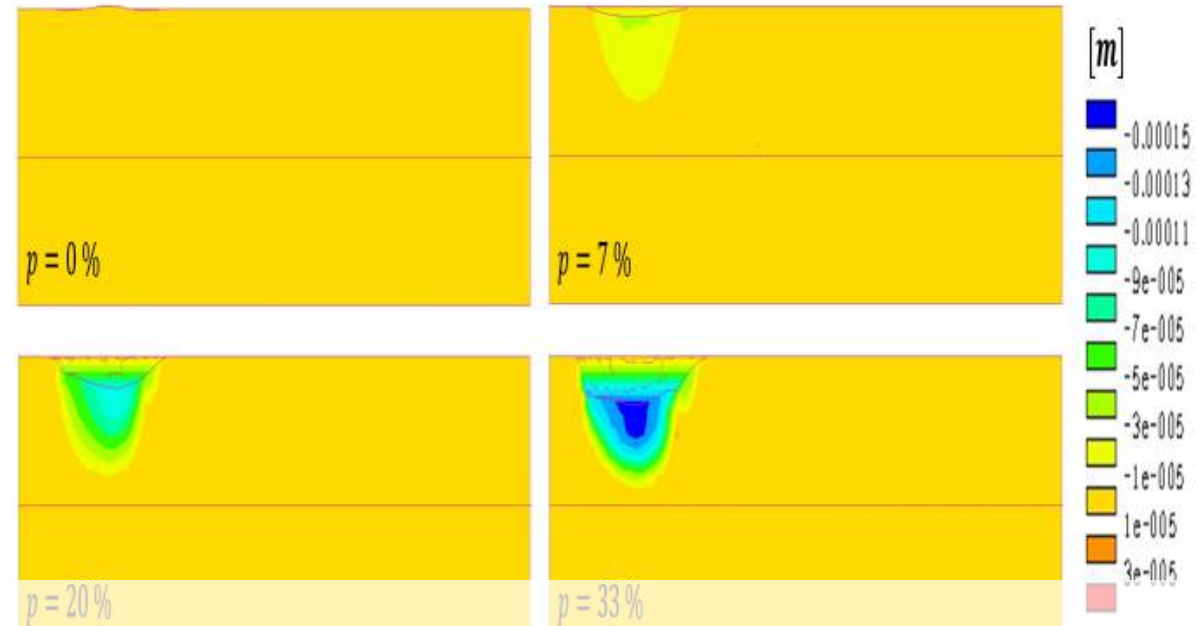

Figure 6: Shrinkage of the powder layer $\Delta e$ after melting (displacement $U_{z}$ at $t=2.4 \mathrm{~ms}$ ) for different powder layer porosities.
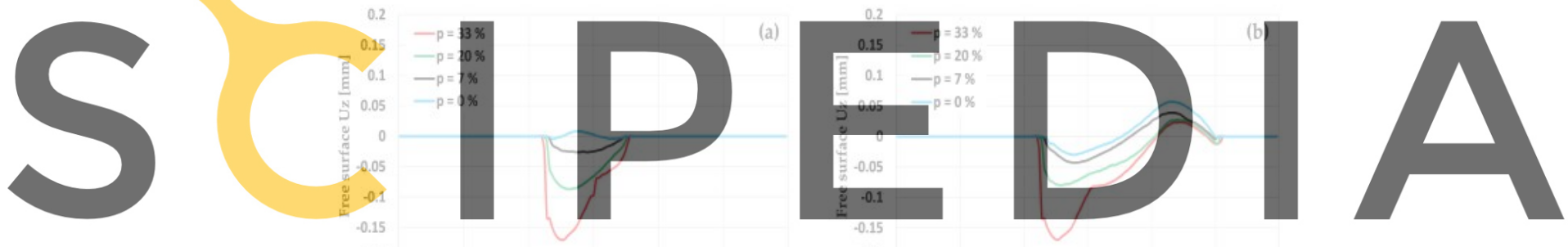

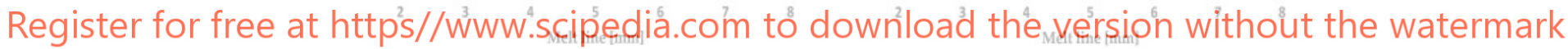

Figure 7: Free surface evolution along the melt line: (a) $t=3 \mathrm{~ms}$, (b) $t=9 \mathrm{~ms}$
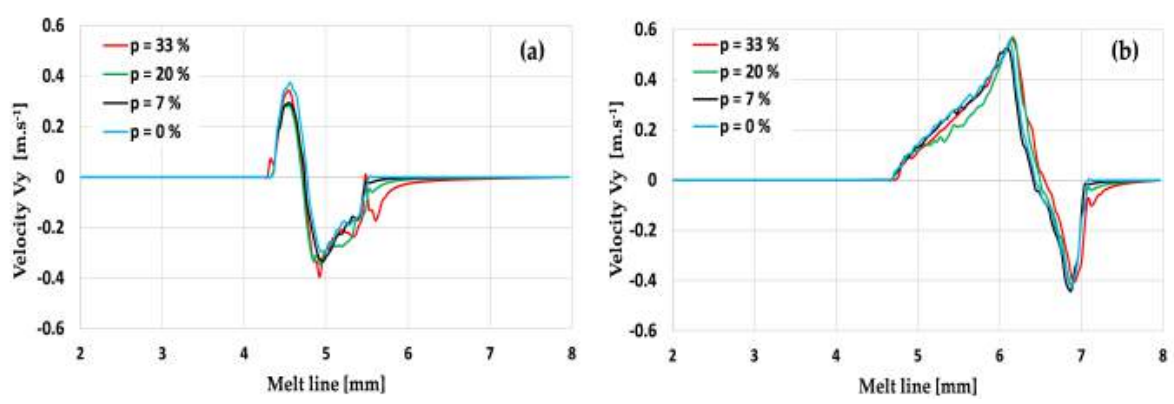

Figure 8: Velocity evolution along the melt line: (a) $t=3 \mathrm{~ms}$, (b) $t=9 \mathrm{~ms}$. 

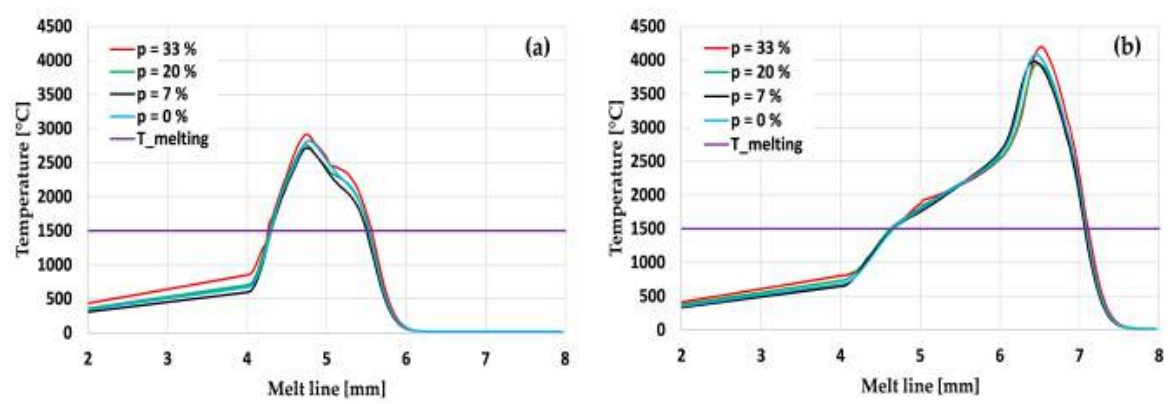

Figure 9: Temperature evolution along the melt line:(a) $t=3 \mathrm{~ms}$, (b) $t=9 \mathrm{~ms}$.

\section{CONCLUSIONS}

In order to simulate the melting of a powder bed, a new numerical approach was proposed. It is based on a fluid formulation that allows us to minimize the computation time. This approach allows taking into account the molten pool formation and the variation of the thermophysical properties (density, thermal conductivity, and specific heat) according to the material state (powder or compact). The variation of the density according to the material state has allowed simulating the powder layer shrinkage after its melting.

As an application, a 3D thermo-fluid simulation of the melting of a powder bed was carried out. The numerical results have shown that the powder laye the free surface evolution. and the temperature were not ver The proposed approach can be improved in order to vaporization, spatter, etc.)
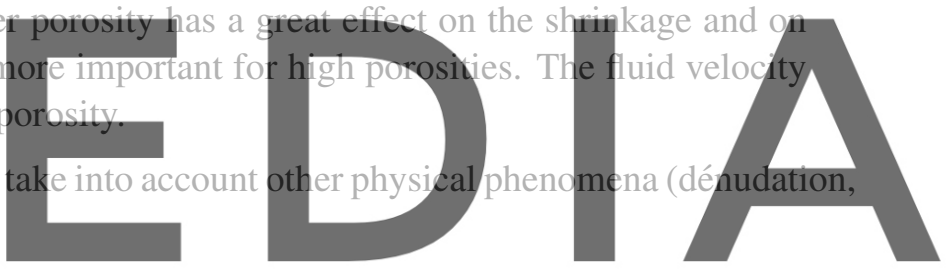

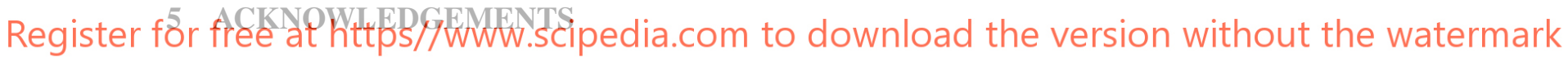

The authors would like to thank ESI Group for funding this study. 


\section{REFERENCES}

[1] Y. Saadlaoui, Julien Sijobert, M. Doubenskaia, P. Bertrand, E. Feulvarch, and J.-M. Bergheau, Experimental Study of Thermomechanical Processes: Laser Welding and Melting of a Powder Bed. Crystals, Vol. 10 (4),pp. 246, 2020.

[2] Y. Saadlaoui, E. Feulvarch, A. Delache, J.-B. Leblond, and J.-M. Bergheau, A new strategy for the numerical modeling of a weld pool. Comptes Rendus Mécanique, Vol. 346 (11), pp. 999-1017, 2017.

[3] Y. Saadlaoui, A. Delache, E. Feulvarch, J.-B. Leblond, and J.-M. Bergheau, New strategy of solid/fluid coupling during numerical simulation of thermo-mechanical processes. Journal of Fluids and Structures, Vol. 99, pp.103161, 2020.

[4] C. Körner, E. Attar, and P. Heinl, Mesoscopic simulation of selective beam melting processes. Journal of Materials Processing Technology, Vol. 211 (6), pp. 978-987, 2011.

[5] Z. Gan, G. Yu, X. He, and S. Li, Numerical simulation of thermal behavior and multicomponent mass transfer in direct laser deposition of Co-base alloy on steel. International Journal of Heat and Mass Transfer, Vol. 104, pp. 28-38, 2017.

[6] Q. Chen, G. Guillemot, C.-A Gandin, and M. Bellet, Three-dimensional finite element thermomechanical modeling of additive manufacturing by selective laser melting for ceramic materials. Additive Manufacturing, Vol. 16, pp. 124-137, 2017.

[7] J. -B. Leblond, H. Amin El Sayed, and J.-M. Bergheau, Mesoscopic On the incorporation of surface tension in finite-element calculations. Comptes Rendus Mécanique, Vol. 341(11-12), pp. 770-775, 2013.

[8] D.N. Arnold, F. Brezzi, M. Fortin, A stable finite for the stokes equations. Calcolo, 21 (4), pp. 337-344, 1984.

[9] J. Goldak, A. Chakravarti, M. Bibby, A New Finite Element Model for Welding Heat Sources, Mettallurgical Transactions B, Vol. 15 (2), pp. 299-305, 1984.

[10] X.S. Gao, C.S. Wu, S.F. Goecke, and H. Kugler, Numerical simulation of temperature field, fluid flow and weld bead formation in oscillating single mode laser-GMA hybrid welding. Journal of Materials Processing Technology. Vol. 242, pp. 147-159, 2017. 